\title{
A sexualidade e os aspectos influenciadores na perspectiva de estudantes universitários
}

\author{
Sexuality and influential aspects from the perspective of university students \\ Sexualidad y aspectos influenciales desde la perspectiva del estudiante universitario
}

Recebido: 31/08/2021 | Revisado: 07/09/2021 | Aceito: 09/09/2021 | Publicado: 12/09/2021

\author{
Débora Fernanda Sousa Marinho \\ ORCID: https://orcid.org/0000-0002-0158-4381 \\ Universidade do Estado do Rio de Janeiro, Brasil \\ E-mail: deborauerj.enf@gmail.com \\ Thelma Spindola \\ ORCID: https://orcid.org/0000-0002-1785-5828 \\ Universidade do Estado do Rio de Janeiro, Brasil \\ E-mail: tspindola.uerj@gmail.com \\ Rômulo Frutuoso Antunes \\ ORCID: https://orcid.org/0000-0003-2800-5295 \\ Instituto Nacional de Câncer, Brasil \\ E-mail: romulofantunes@gmail.com \\ Cristiane Maria Amorim Costa \\ ORCID: https://orcid.org/0000-0003-1089-2092 \\ Universidade do Estado do Rio de Janeiro, Brasil \\ E-mail: cmacosta1964@gmail.com \\ Raquel Ramos Woodtli \\ ORCID: https://orcid.org/0000-0002-6418-1037 \\ Instituto Fernandes Figueira, Brasil \\ E-mail: raquel_rrw@hotmail.com \\ Nathália dos Santos Trindade Moerbeck \\ ORCID: https://orcid.org/0000-0002-0405-0764 \\ Universidade do Estado do Rio de Janeiro, Brasil \\ E-mail: nathaliatrindade_15@hotmail.com
}

\begin{abstract}
Resumo
Objetivo: Compreender a percepção de jovens universitários sobre sexualidade, e descrever os fatores influenciadores da sexualidade na concepção dos estudantes. Método: Estudo descritivo, qualitativo, realizado numa universidade pública. Participaram 27 universitários, de ambos os gêneros, idades entre 18-29 anos. Foram realizados três grupos focais onde se discutiram aspectos sociais, culturais, condutas sexuais e prevenção de infecções sexualmente transmissíveis. $\mathrm{Na}$ análise dos dados empregou-se a técnica de análise de conteúdo, emergindo três categorias. Resultados: A sexualidade na ótica dos jovens e fatores influenciadores da sexualidade na perspectiva dos universitários foram categorias com maior representatividade (54\%). O conceito de sexualidade está relacionado ao autorreconhecimento, atração física, desejo e liberdade de expressão, e a influência da cultura e religiosidade na sexualidade dos jovens. Conclusão: O comportamento sexual sofre influência de aspectos culturais, sociais, religiosos, relacionamentos interpessoais e afetivos, sendo modulado pelo grupo de pertença dos jovens.
\end{abstract}

Palavras-chave: Sexualidade; Adulto jovem; Comportamento sexual; Religião; Cultura.

\begin{abstract}
Objective: To understand the perception of young university students about sexuality, and to describe the influencing factors of sexuality in the students' conception. Method: A descriptive, qualitative study was conducted at a public university. Twenty-seven university students, of both genders, aged 18-29 years, participated. Three focus groups were conducted to discuss social, cultural aspects, sexual behavior and prevention of sexually transmitted infections. In the data analysis, the content analysis technique was used, emerging three categories. Results: Sexuality from the perspective of young people and factors influencing sexuality from the perspective of university students were categories with greater representativeness $(54 \%)$. The concept of sexuality is related to self-recognition, physical attraction, desire and freedom of expression, and the influence of culture and religiosity on the sexuality of young people. Conclusion: Sexual behavior is influenced by cultural, social, religious, interpersonal and affective relationships, being modulated by the group of belonging of young people.
\end{abstract}

Keywords: Sexuality; Young adult; Sexual behavior; Religion; Culture. 


\begin{abstract}
Resumen
Objetivo: Comprender la percepción de los jóvenes universitarios sobre la sexualidad, y describir los factores que influyen en la concepción de la sexualidad de los estudiantes. Método: Se trata de un estudio descriptivo y cualitativo realizado en una universidad pública. Participaron 27 estudiantes universitarios, de ambos sexos, con edades comprendidas entre los 18 y 29 años. Se realizaron tres grupos de discusión en los que se debatieron aspectos sociales y culturales, comportamientos sexuales y prevención de infecciones de transmisión sexual. En el análisis de datos se utilizó la técnica de análisis de contenido, surgiendo tres categorías. Resultados: La sexualidad desde la perspectiva de los jóvenes y los factores que influyen en la sexualidad desde la perspectiva de los estudiantes universitarios fueron las categorías con mayor representatividad (54\%). El concepto de sexualidad está relacionado con el reconocimiento de sí mismo, la atracción física, el deseo y la libertad de expresión, y la influencia de la cultura y la religiosidad en la sexualidad de los jóvenes. Conclusão: Se concluye que el comportamiento sexual está influenciado por aspectos culturales, sociales, religiosos, relaciones interpersonales y afectivas, siendo modulado por el grupo al que pertenecen los jóvenes.
\end{abstract}

Palabras clave: Sexualidad; Adulto joven; Conducta sexual; Religión; Cultura.

\title{
1. Introdução
}

A sexualidade é compreendida como um aspecto fundamental do ciclo de vida humano, envolve desejos e práticas relacionados ao prazer, aos sentimentos, à saúde e ao desempenho de liberdade, na qual o corpo físico é somente um alicerce para a construção histórica e de identidade que envolve outras construções como individualidade, satisfação sexual e apetite erótico (Zerbinati \& Bruns, 2017).

A experiência da sexualidade é um processo de ensaio individual e absorção cultural do grupo ao qual se pertence, onde o mecanismo de desenvolvimento da sexualidade se manifesta pela transformação biológica, principalmente na adolescência e juventude, ocasião em que essas mudanças se expressam com mais intensidade (Ferrari \& Nascimento, 2018).

A sexualidade precisa ser compreendida como uma produção cultural e social, na qual as formas de viver nossos prazeres e desejos não são proporcionadas pela natureza, visto que há uma complexa combinação de sentidos, representações, e atribuições que efetivamente vão constituí-la. A individualidade sexual é um conjunto de produtos do desenvolvimento, composta por fatores históricos, culturais, sociais, e exercida através de mitos, tabus e relações de poder, ou seja, as práticas sexuais presentes na juventude são conduzidas de acordo com as relações interpessoais e individuais (Teixeira, 2015; Teixeira, 2016).

Para os jovens, a esfera da sexualidade se apresenta em um campo de experiências, comunicações, sentimentos, vivências e descobertas, na construção da capacidade de tomar decisão, de preferências e da certificação de identidade. A sexualidade se salienta no âmbito da busca pela autonomia de práticas e projetos, sendo desempenhada de maneira única e com ansiedade pela juventude (Ferrari \& Nascimento, 2018).

Assim sendo, é necessário discutir as peculiaridades e aspectos influenciadores do grupo jovem, tendo em vista a compreensão do comportamento social que ocupam e as inúmeras pluralidades que enfrentam nessa faixa etária, para poder subsidiar as perspectivas e anseios da sexualidade. Esta pesquisa tem o objetivo de compreender a percepção de jovens universitários sobre a sexualidade, e descrever os fatores influenciadores da sexualidade na concepção dos estudantes.

\section{Metodologia}

Trata-se de um estudo descritivo com abordagem qualitativa fundamentada na teoria dos roteiros sexuais. Este estudo está integrado à pesquisa "Sexualidade e vulnerabilidade dos jovens em tempos de Infecções Sexualmente Transmissíveis", vinculada ao Programa de Pós-Graduação de Enfermagem da Universidade do Estado do Rio de Janeiro.

O cenário do estudo foi uma instituição de ensino superior pública, localizada na cidade do Rio de Janeiro. Participaram 27 universitários, sendo 12 do gênero masculino e 15 do feminino, considerando o interesse em investigar estudantes de ambos os gêneros e contemplando a homogeneidade dos grupos focais. $\mathrm{O}$ estudo adotou como critérios de 
inclusão foram: ser aluno regularmente matriculado em curso de graduação da universidade e idade entre 18-29 anos. Foram excluídos alunos ausentes, no período de coleta de dados, por motivo de trancamento de matricula ou adoecimento.

Foi selecionada uma amostra por conveniência dos universitários de ambos os gêneros presentes no campo da pesquisa na ocasião da coleta de dados. Os dados foram coletados em 2018, com emprego da técnica de GF, havendo a participação de nove estudantes de ambos os gêneros a cada encontro. Colomé et al., (2015) salientam que o GF se trata de uma entrevista em grupo, informal, em que o diálogo e a problematização fazem parte da mesma técnica. Como estratégia para o alcance dos objetivos do estudo, empregou-se um roteiro com temas discutidos com os estudantes, a saber: sexualidade; condutas sexuais/gênero e prevenção de IST, que tinham aderência ao objeto deste estudo. Os GF duraram cerca de 120 minutos, foram conduzidos por uma moderadora e duas observadoras. Ao final do terceiro GF percebeu-se a saturação das informações, ou seja, os dados discursivos se repetiam e não agregavam novos esclarecimentos ao objeto estudado, momento que se encerrou a coleta.

Os conteúdos discursivos dos GF foram transcritos na íntegra para a construção do corpus, que foi formatado de modo a tornar o material compreensível, foram suprimidos do texto analisado os vícios de linguagem, expressões não verbais e palavras de uso coloquial inadequada para a pesquisa científica.

Os dados foram analisados com emprego da técnica de análise de conteúdo, no modelo análise temático-categorial proposto por Bardin (2011) e sistematizado por Oliveira (2008). Para Bardin (2011), a análise de conteúdo pode ser entendida como um recurso empírico, cujo propósito é esclarecer o crítico. A autora apresenta como métodos de análise a organização, codificação, categorização, influência e informatização (Bardin, 2011).

Os resultados dos dados analisados foram expressos em categorias, que contém temas identificados no conteúdo das falas dos participantes. Nesse processo foram reveladas duas categorias discursivas com aderência aos objetivos deste estudo, a saber: 1- A sexualidade na ótica dos jovens; 2- Fatores influenciadores da sexualidade na perspectiva dos universitários.

Foram respeitadas todas as normas e diretrizes para estudos envolvendo seres humanos conforme a Resolução 466/2012 da Comissão Nacional de Ética em Pesquisa. A investigação foi aprovada pela Comissão de Ética e Pesquisa da instituição sede do estudo, com parecer 902543 e todos os participantes fizeram a leitura e assinaram o Termo de Consentimento Livre e Esclarecido.

\section{Resultados e Discussão}

Os universitários participantes do estudo ( $n=27)$ tinham a seguinte caracterização: idades entre 18 anos e 24 anos $(\mathrm{n}=24)$; situação marital - solteiro/sem namorado $(\mathrm{n}=16)$; tem namorado $(\mathrm{n}=9)$; sem filhos $(\mathrm{n}=26)$; residiam com os pais$(n=23)$; estudante da área da saúde ( $n=10)$, humanas $(n=9)$, exatas $(n=8)$.

$\mathrm{Na}$ análise discursiva dos dados emergiram três categorias, contudo neste artigo serão apresentadas duas que representam 55\% do corpus analisado: A sexualidade na ótica dos jovens, que concentra $20 \%$ do corpus e Fatores influenciadores da sexualidade na perspectiva dos universitários, com $35 \%$.

Categoria 1: A sexualidade na ótica dos jovens

Essa categoria concentra sete unidades de significação e representa $20 \%$ do total do corpus analisado. No entendimento dos participantes o conceito de sexualidade está relacionado ao autorreconhecimento, atração física, desejo e liberdade de expressão.

Sexualidade [...] é a expressão de um desejo íntimo da pessoa, como expressa, o que sente por outro ser humano. (Participante 1,19 anos) 
[...] como você se atrai por outra pessoa. Sexualidade sempre envolve você e outra pessoa [...]. Sempre determinado por quem você se atrai. (Participante 4, 21 anos)

É como você se reconhece, como mulher, como homem, às vezes tem mulheres que se reconhecem como homem. (Participante 9, 21 anos)

Sexualidade é a forma expressa do gosto, não tem relação com a biologia, propriamente dita. Acho que sexualidade é como você se vê no mundo. (Participante 6, 21 anos)

[...] para mim, é uma coisa que pode ser mudada. Não necessariamente você é do jeito que nasceu. (Participante 10, 19 anos)

Na compreensão dos jovens o conceito de sexualidade está associado ao sexo, desejo, atração física, sendo entendida como expressão dos desejos íntimos das pessoas. O estudo feito por Silva et al. (2019) com o objetivo de compreender as percepções dos estudantes de enfermagem acerca da sexualidade, obteve os dados semelhantes aos apresentados nesta pesquisa. Dessa forma, constatou-se que os conceitos relatados pelo público entrevistado sobre a sexualidade referem-se aos comportamentos sexuais, ao amor, à intimidade, ao componente fundamental das relações e paixões sexuais, ao prazer e, também, à saúde reprodutiva.

Assim, a sexualidade abrange diversas dimensões do ciclo de vida humano como gênero, orientação e identidade sexual, emoção, reprodução e amor. E é manifestada e vivida individualmente e de distintas formas, por exemplo, por meio dos desejos, fantasias, pensamentos, valores, ações, relacionamentos (World Health Organization, 2017; Silva et al., 2019; Alvez et al., 2017). Está associada ao cenário social dos valores, dos comportamentos, dos desejos, das crenças, dos relacionamentos interpessoais e da identidade, dando-se em novas conjunturas ao longo do tempo. Por conseguinte, ela abarca um conceito diferente de sexo, que alude à objeção biológica guiada pelo aparelho reprodutivo (Alvez et al., 2017).

Sexualidade, no entanto, não se mescla com o instinto, com o parceiro ou com a fusão dos órgãos genitais. Ela é multifacetada e ultrapassa a necessidade fisiológica e tem relação com a caracterização do desejo. Mesmo que receba a influência biológica, não se limita somente aos aspectos genitais, mas também a como o indivíduo entende e vivencia os enredos afetivos e sexuais ao longo da existência. Assim, os grupos sociais, o tempo e fatores históricos contribuem para a sua construção (Senem \& Caramaschi, 2017).

A composição cromossômica da pessoa humana compõe a primeira característica para diferenciar os dois sexos. Como resultado, a fisiologia e anatomia das estruturas sexuais estabelecem macho e fêmea. A identidade de gênero que caracteriza a feminilidade e a masculinidade recebe influência de vários fatores, e está relacionada à autodefinição (Teixeira, 2016; Aufranc, 2018).

Gênero é entendido como a constituição social e cultural das funções práticas de ser homem e mulher, influenciado por hábitos, crenças e tempo. Dessa maneira, determinados comportamentos são esperados de homens e mulheres em todas as culturas, contudo podem apresentar distinções. O conceito de gênero não está associado somente aos comportamentos sexuais já esperados, tendo em vista que a orientação sexual é entendida como a busca de relacionamentos afetivo-sexuais por indivíduos de gêneros diferentes, do mesmo gênero ou ambos (Teixeira, 2016; Lusey et al., 2017).

Pode-se perceber, então, a forte influência do contexto histórico e dos papeis sociais nos relacionamentos afetivosexuais (Nogueira \& Muzzeti, 2017). Papeis de gênero são ensinados desde a infância e estabelecem as diferenças entre meninas e meninos. As condutas femininas e masculinas são incentivadas e reforçadas pelos pais, pelas instituições sociais e pessoas mais próximas (De Bolle et al., 2015).

Nas falas dos participantes percebe-se que os jovens reconhecem a sexualidade vinculada à identidade de gênero, à orientação sexual, à identidade biológica, ou seja, a uma identidade fluída que pode alterar e diversificar entre a identidade de gênero. As representações sobre sexualidade revelam uma nova percepção da liberdade sexual. Dessa forma, percebe-se que a 
sexualidade tem em si a vivência do sentido, sendo um aspecto dinâmico, dialético, histórico e processual. Além de transitar pelas dimensões da reprodução, da prática sexual e do prazer (Senem \& Caramaschi, 2017).

Entender que a sexualidade é um processo contínuo de transformação é fundamental para distinguir os modos de vivenciá-la e reconhecer suas variâncias no contexto histórico. Os padrões e normatizações estabelecidos influenciam a expressão e interpretação crítica dos comportamentos. É válido compreender os mais diversos fatores que contribuíram, ao longo do tempo, para estruturar o modo como a sociedade visualiza a sexualidade. A sexualidade, portanto, compreende várias dimensões como a social, histórica, cultural e biológica (Senem \& Caramaschi, 2017).

Categoria 2: Fatores influenciadores da sexualidade na perspectiva dos universitários

Essa categoria concentra 15 unidades de significação e representa $35 \%$ do corpus de análise. Aborda a perspectiva dos estudantes sobre os fatores que influenciam a sexualidade dos jovens.

[...] têm muitos pais que tratam o sexo como um tabu [...] todo mundo faz sexo, caso contrário não estaríamos aqui. [...] os pais não conversam [...] com os filhos. Participante 7, 29 anos).

[...] vários fatores interferem [na sexualidade], não só a questão cultural, mas também a religiosa [...] conceitos são mantidos até os dias de hoje. Tenho amigas que têm vontade de transar com o namorado, mas não fazem por questões religiosas. Sexo só é permitido após o casamento. [...] Essas meninas até praticam, mas escondido [...]. A igreja e a família não podem saber. (Participante 8, 24 anos)

O comportamento do homem é muito mais livre. Qualquer coisa que ela [mulher] faz a sociedade já cai em cima [critica]. [...] ela faz muitas coisas, mas guarda para ela. O homem vai à rodinha de amigos e explana para todos. Ele é visto como o pegador. (Participante 1, 19 anos).

[...] há uma diferença [no comportamento sexual] entre o homem e a mulher. [...] Ele é mais aberto às experiências sexuais [...] A mulher desde cedo é ensinada que o sexo é por amor, por sentimento. Eu acho que devido à cultura brasileira. Por isso, a mulher se trava um pouco. (Participante 9, 21 anos).

[...] Eu sinto que tem um controle [social] muito maior sobre o corpo da mulher e [...] se você quer se expressar, quer viver a sua vida, é muito mais difícil [...]. (Participante 18, 22 anos).

No tocante aos fatores que interferem na sexualidade dos jovens, descrito na categoria 2, percebeu-se a influência dos aspectos culturais e geográficos nos comportamentos sexuais. Sabe-se que os mecanismos da sexualidade são permeados pela intenção, escolha e, também, pela dimensão cultural. Assim, a cultura de diferentes sociedades e o modo como vivenciam os valores morais influenciam os comportamentos sexuais (Senem \& Caramaschi, 2017; Somefun, 2019).

Nas falas dos estudantes, percebeu-se a insuficiência (ou inexistência) de comunicação entre pais e filhos acerca da sexualidade. A falta de diálogo sobre essa temática denota a existência de tabus e inibições entre pais e filhos. Os pais são as pessoas mais próximas dos jovens, e esperava-se que fossem os primeiros a conversar com os filhos a respeito do sexo e sexualidade, contudo essa não é uma prática habitual nas famílias. Muitos pais acreditam que conversar sobre esse tema estimula as práticas sexuais. Estudo de Silva \& Castro (2018), a respeito dos diálogos sobre sexualidade entre pais e filhos adolescentes, revela que a ausência de informações sobre sexualidade vem de gerações anteriores, e contribui para que os pais não conversem com os filhos a respeito desse tema. Já os filhos se ressentem da falta desse diálogo com seus pais.

Sabe-se que o diálogo sobre as práticas sexuais torna a iniciação sexual precoce menos frequente, e reduz o número de parceiros sexuais. A premissa do sexo escondido, do prazer impróprio e as manifestações sexuais nem sempre foram cobertas e reprimidas na cultura social. Esse fato ocorreu no período da Reforma Protestante, quando a linguagem relacionada à sexualidade foi implementada como pecado. O catolicismo começou, então, a enxergar a sexualidade como algo ruim, mas que 
deveria ser consentido no casamento pela necessidade da procriação. Esse fato ocorreu devido à contribuição da filosofia grega gnóstica que engrandecia o espírito acima do corpo (Kösterbenger, 2015; Senem \& Caramasch, 2017; Somefun, 2019).

Estudo de Spindola et al. (2020) sobre a iniciação sexual dos jovens e o diálogo acerca da sexualidade evidenciou a influência da religião, principalmente pentecostais e protestantes, nas práticas sexuais dos jovens. Nos relatos dos jovens percebeu-se que o sexo é entendido como uma manifestação do desejo físico, mas é influenciado pelos dogmas religiosos. As universitárias revelaram, ainda, que a sociedade e a cultura do seu grupo de pertença, e para alguns a própria religião, influenciam suas condutas, especialmente as práticas sexuais (Spindola et al., 2020). Sabe-se que existem situações, nas quais a pessoa não verbaliza (ou esconde) suas condutas para evitar o confronto com a crença religiosa, conforme a fala da participante 8 nesta pesquisa sinalizou.

As condutas, os pensamentos, valores sociais e culturais dos indivíduos estão associados à religião e religiosidade. Apesar da participação e envolvimento em algum grupo religioso não ser prática, a religiosidade subjetiva é considerada parâmetro para manter as crenças culturais (Lassiter et al., 2017; Busin, 2011). Estudo de Bodanese \& Martins (2017) revela que a cultura ocidental, em especial a judaico-cristã, apresenta dificuldades em dialogar sobre sexualidade. Esse déficit é observado desde o ensino nas escolas, visto que, é pautado na anatomia humana e em ações para prevenir doenças, sendo biologicamente padronizado, enquanto a sexualidade continua sendo um tabu.

Nos relatos dos participantes percebe-se que a religião se entrelaça com aspectos culturais arraigados na sociedade. Entendida como parâmetro para os comportamentos sociais, relacionamentos interpessoais e para estabelecer a identidade cultural e a socialização, dentro ou fora das instituições religiosas, assevera Smith (2003). Assim, as normas religiosas tendem a formatar o comportamento sexual (Lassiter et al., 2017).

No estudo de Oliveira (2018), grupos divergentes possuem discurso pautado na inclusão das minorias sexuais, fazendo com que as mesmas possam exercer a sua religiosidade, ainda que haja o predomínio do conservadorismo no contexto brasileiro. Nas descrições dos estudantes universitários, os modelos inculcados pelas crenças religiosas influenciam a sexualidade e as práticas sexuais dos grupos sociais. Sabe-se, contudo, que esses dogmas e normas nem sempre são seguidos por todos. No entender de Coutinho \& Ribeiro (2014), alguns conceitos, uma vez inseridos no contexto do jovem - seja por meio dos comportamentos culturais mais vistos ou por algum contato com a religião - já são suficientes para ter influência em suas condutas sexuais. Por outro lado, na atualidade apesar do jovem vivenciar maior liberdade sexual, muitos escondem sua sexualidade no ambiente familiar ou religioso, o que pode ser um agravo para a sua saúde sexual.

Não obstante, a proximidade com alguma religião não caracteriza o indivíduo como religioso. Compreende-se por religião um agrupamento de rituais, códigos e crenças morais partilhados por seus fiéis. Já a religiosidade é entendida como a prática e cumprimento das diretrizes e preceitos de uma determinada religião (Coutinho \& Ribeiro, 2014; Silva, Passos, \& Souza, 2015; Couto et al., 2018). Ainda no aspecto da religiosidade, dois modelos são existentes: a religiosidade intrínseca e a extrínseca. Quanto à primeira, o indivíduo coloca a religião no centro da sua vida integrando modelos culturais e conceitos morais, que agregam sentido à vida. Já no segundo modelo, a religião está relacionada a um complexo de crenças e atividades, sendo uma forma de trazer consolo e sociabilidade (Silva, Passos, \& Souza, 2015).

Ser religioso não quer dizer ter grande religiosidade, mas significa que o indivíduo pertence e/ou acredita em uma fé. A religiosidade tem relação com a prática e atitude referente à religião, como ler materiais sagrados, frequentar reuniões/cultos e participar das atividades religiosas, independentemente da fé (Francis et al., 2019). Na perspectiva dos referidos autores a interferência da religiosidade vai além dos aspectos relacionados à sexualidade e comportamento sexual, sendo significativa quanto ao uso de álcool ou drogas e na iniciação sexual precoce. Acreditam, ainda, que quanto maior a religiosidade menor o uso do álcool, do tabaco e das drogas, além de reduzir as taxas de ações agressivas entre as pessoas (Francis et al., 2019). 
$\mathrm{Na}$ concepção dos participantes, existem comportamentos sexuais diferenciados entre homens e mulheres. Espera-se que o homem tenha mais experiências sexuais, sendo essa prática valorizada. No que concerne às mulheres, é esperado um comportamento mais discreto, menor exposição das condutas sexuais, não sendo uma prática "aprovada" quando a menina demonstra uma conduta sexual semelhante aos homens. Percebe-se que as mulheres, ainda, são mais pressionadas que os homens para não manter relações sexuais antes do casamento. A religião tem influenciado o comportamento humano desde os primórdios da humanidade, e influencia os hábitos e costumes de todas as culturas. A sexualidade e o comportamento sexual da humanidade no passado foram determinados pela religião, e muitas dessas determinações, ainda, estão presentes na moralidade dos dias atuais. A sexualidade hoje, ainda, é estruturada em valores e crenças que determinam o que é proibido e o que deve ser resguardado (Duarte, 2017).

Assim, existe um controle sobre o corpo, o modo como deve se comportar. Esse modelo é denominado de biopoder e objetiva moderar a sexualidade por meio de argumentos proibitivos e intercessões sociais, principalmente do corpo feminino. Nesse modelo de controle, as religiões são fortemente adeptas através das normas e dogmas. Dessa maneira, a virgindade e a castidade, assim como prática sexual dentro do casamento, configuraram o sexo com consenso aceito pelas religiões. E, nesse contexto, foi pactuado que esses comportamentos favoreceriam a prevenção de doenças (Couto et al., 2018).

Entretanto, essas diretrizes impostas às mulheres e aos homens tendem a colocar em risco a saúde. Lusey et al. (2017) afirmam que os jovens acreditam na representação da masculinidade, sendo natural praticar inúmeras relações sexuais e não se preocupar com a proteção, além de esperar que prevenção da gravidez seja um dever da mulher. Na concepção de Aufranc (2018), à mulher coube o papel materno e o lar, sendo moldada a imagem da mulher comprometida com o marido e filhos. Por conseguinte, sua vida sexual ficaria restrita à procriação. O prazer sexual e as experimentações do sexo foram concedidos ao homem. A mulher mãe jamais deveria sentir prazer, já que o pensamento difundido era que o instinto materno deveria abolir o desejo sexual.

Embora a família seja influenciada por meios de controle como a mídia, a economia, a religião entre outros, possui grande valor para a estruturação sócio-histórica dos papeis de homens e mulheres na sociedade. O contexto familiar ocupa o papel de influenciado e influenciador para o aspecto cultural, assim, o núcleo familiar age como uma instituição controladora que impõe aos membros como devem ser comportar. As informações sobre o certo e errado, aceitável ou não são iniciadas e moldadas nesse ambiente (Landim \& Borsa, 2019)

As especificações e demarcações das diferenças sexuais são apresentadas e construídas, nesse cenário. A mulher e o homem foram efetuando as funções estabelecidas, como o cuidado, a organização e o controle. As mudanças nos papeis se devem às evoluções da sociedade e da industrialização, já que a dedicação exclusiva da mulher aos filhos foi dividida, muitas vezes, com as babás e escolas integrais. Para analisar os comportamentos sexuais e sociais, devem-se considerar essas transformações familiares, dado que as construções socioculturais são influenciadas por mecanismos históricos e culturais. A implantação dos conceitos sobre comportamento, entretanto, interfere diretamente na perspectiva e entendimento do jovem acerca da conduta sexual e suas manifestações (De Bolle et al., 2015).

\section{Conclusão}

Conclui-se que os jovens compreendem a sexualidade em uma visão plural, que assume contornos diferenciados conforme a cultura e religiosidade das pessoas. Acreditam que a sexualidade é influenciada pelo ambiente social, pela família e preferência individual de cada pessoa. Na concepção dos participantes, o conceito de sexualidade está relacionado com sexo, atração física e ao desejo sexual, além de estar vinculado à identidade de gênero, orientação sexual e identidade biológica. 
Pode-se perceber que os estudantes têm conhecimento sobre esses temas, porém, mostram ambiguidade nas definições dos mesmos. Acreditam que é importante ter liberdade para assumir a sexualidade perante a sociedade, mas que a vivência da sexualidade pode acarretar discriminação, como ocorre com pessoas que assumem a homossexualidade.

No contexto da influência da religiosidade nas práticas sexuais, embora ela desempenhe uma função restritiva na vida das pessoas, muitas vezes pode agir como protetora dos comportamentos sexuais. Jovens religiosos ou que frequentam alguma religião são mais propensos a adiar a prática sexual, consomem menos álcool e outras drogas, não estão envolvidos em práticas sociais punitivas e, por consequência, têm menos comportamentos que acarretam riscos para a sua saúde. Sabe-se que qualquer civilização ou cultura tem seus mecanismos e formas para entender e dar sentido à vida e regular as ações coletivas e individuais. A maneira como o jovem vislumbra a vida é influenciada pela cultura e religiosidade. E isso pode favorecer a formação de atitudes resilientes, que irão contribuir para a manutenção de sua saúde mental e emocional.

A religiosidade, então, pode favorecer a busca de relacionamentos interpessoais mais saudáveis e duradouros, com respeito e cuidado para com o outro. São estimulados a desenvolver laços familiares e sociais mais sadios, e a reduzir comportamentos violentos e maléficos à sociedade.

Considerando que os jovens são a parcela da população mais exposta aos agravos de saúde, acredita-se que a informação e o esclarecimento desse grupo pelos pais, educadores e profissionais de saúde são relevantes e devem ser estimulados para minorar comportamentos sexuais de risco para a saúde sexual do grupo. Nesse sentido, sugere-se a realização de novas investigações acerca da importância do diálogo entre pais e filhos como instrumento de promoção da saúde no escopo familiar sejam estimulados, a fim de se obter maiores evidências científicas.

\section{Referências}

Alvez, B., Borges, M. G., Fontoura, V. L., \& D'Eça, G. N. (2017). Sexual profile of university student. Rev Bras Promoção Saúde. 30(4), 1643-64. https://pesquisa.bvsalud.org/portal/resource/pt/biblio-876527

Aufranc, A. B. (2018). Expressões da sexualidade: um olhar junguiano. Junguiana, 36(1), 37-48. http://pepsic.bvsalud.org/scielo.p hp?script=sci_arttext\&pid=S010308252018000100007\&lng=pt\&nrm=iso

Bardin, L. (2011). Análise de conteúdo. (70a ed.).

Bodanese, G. R., \& Martins, M. E. (2017). Sexualidade, comportamento sexual e cultura - reflexões e articulações teóricas. Psicologia.pt, 1-12. https://www.psicologia.pt/artigos/textos/A1328.pdf

Busin, V. M. (2011). Religião, sexualidades e gênero. REVER - Revista de Estudos da Religião. 11(1). https://revistas.pucsp.br/rever/article/view/6032

Colomé, J. S. et al (2015). Metodologias da pesquisa para a enfermagem e saúde: da teoria à prática. In: Costenaro, R. G. S., \& Lacerda, M. R. Grupo focal como técnica de coleta de dados: questões teóricas e práticas. 433-450.

Coutinho, R. Z., \& Ribeiro, P. M. (2014). Religião, religiosidade e iniciação sexual na adolescência e juventude. Rev Bras Est Pop, 31(2), 333-365. https://www.scielo.br/pdf/rbepop/v31n2/a06v31n2.pdf

Couto, P. L. S., Paiva, M. S., Oliveira, J. F., Tosoli, A. M. G., Argolo, M. T., \& Teixeira. E. B. (2018). Sexuality and HIV prevention: consensus and dissent of Catholic youths. Investigación y Educación en Enfermería. 36(2), 6-12. http://aprendeenlinea.udea.edu.co /revistas/index.php/iee/article/view/333655/20789617

De Bolle, M., De Fruyt, F., McCrae, R. R., Löckenhoff, C. E., Costa, P. T., et al. (2015). The emergence of sex differences in personality traits in early adolescence: A cross-sectional, cross-cultural study. J Pers Soc Psychol. 108(1), 171-185. https://www.ncbi.nlm.nih.gov/pmc/articles/PMC4327943/

Duarte, A. J. (2017). Religião e comportamento sexual: concepções cristãs sobre sexualidade. Rev Relegens Thréskeia. 6(1), 74-98. https://revistas.ufpr. br/relegens/article/view/54134/34185

Duarte, L. F. D., et al. (2005). Família, Reprodução e Ethos religioso: uma pesquisa qualitativa no Rio de Janeiro. https://www.ces.uc.pt/lab 2004/inscricao/pdfs/painel18/LuizDuarte.pdf

Ferrari, W., Peres, S., \& Nascimento M. (2018). Experimentação e aprendizagem na trajetória afetiva e sexual de jovens de uma favela do Rio de Janeiro, Brasil, com experiência de aborto clandestino. Ciência \& Saúde Coletiva. 23(9), 2937-2950. https://www.scielo.br/pdf/csc/v23n9/1413-8123-csc-23-092937.pdf

Francis, J. M., Myers, B., Nkosi, S., Petersen Williams, P., Carney, T., Lombard, C., Nel, E., \& Morojele, N. (2019). The prevalence of religiosity and association between religiosity and alcohol use, other drug use, and risky sexual behaviours among grade 8-10 learners in Western Cape, South Africa. PLoS ONE. 14(2), 1-20. https://www.ncbi.nlm.nih.gov/pmc/articles/PMC6374069/ 
Köstenberger, A. J. (2015). Deus, casamento e família: reconstruindo o fundamento bíblico. (2a ed.) Vida Nova.

Landim, I., \& Borsa, J. C. (2019). Concepções de famílias: Um estudo sobre as representações gráficas de crianças cariocas. Estud. pesqui. psicol. 19(2), 503521. https://www.e-publicacoes.uerj.br/index.php/revispsi/article/view/44284/30306

Lassiter, J. M., Saleh, L., Starks, T., Grov, C., Ventuneac, A., \& Parsons, J. T. (2017). Race, ethnicity, religious affiliation, and education are associated with gay and bisexual men's religious and spiritual participation and beliefs: Results from the One Thousand Strong cohort. Cultur Divers Ethnic Minor Psychol. 23(4), 468-476. https://www.ncbi.nlm.nih.gov/pmc/articles/PMC5581734/

Lusey, H., San Sebastian, M., Christianson, M., \& Kerstin, E. E. (2017). Factors associated with gender equality among church-going Young men in Kinshasa, Democratic Republic of Congo: a cross-sectional study. Revista internacional para a equidade em saúde. 16(1), 01-11. https://www.ncbi.nlm.nih.gov/pubmed/29228996

Nogueira, N. S., \& Muzzeti, L. R. (2017). A relação amorosa o "ficar" a partir do habitus e da trajetória de vida dos jovens universitários. Humanidades \& Inovação. 4(6),67-77. https://revista.unitins.br/index.php/humanidadeseinovacao/article/view/522

Oliveira, B. A. (2018). As novas guerras sexuais: diferença, poder religioso e identidades LGBT no Brasil. Revista Brasileira de Estudos da Homocultura. 2(1). https://periodicoscientificos.ufmt.br/ojs/index.php/rebeh/article/view/9942

Senem, C. J., \& Caramaschi, S. (2017). Conception of gender and sexuality in the west: origin, history and present. Revista Barbarói. 1(49), 166-189. https://www.heritage.org/gender/report/sex-gender-and-the-originthe-culture-wars-intellectual-history

Silva, B. C., \& Castro, R. D. (2018). Diálogos sobre sexualidade entre pais e filhos adolescentes dentro do contexto familiar. Revista Brasileira de Ciências da Vida. 6(2). http://jornalold.faculdadecienciasdavida.com.br/index.php/RBCV/article/view/611/294

Silva, T. R. F., Fernandes, S. E. T., Alves, N. R., Farias, A. J. A. de, Silva Júnior, J. A. da, \& Santos, S. M. P. dos (2019). Representações dos estudantes de enfermagem sobre sexualidade: entre estereótipos e tabus. Rev. Trab. educ. saúde. 17(2) 1-18. http://www.scielo.br/pdf/tes/v17n2/1678-1007-tes-17-02e0020233.pdf

Silva, S. K., Passos, S. M. K., \& Souza, L. D. M. (2015). Associação entre religiosidade e saúde mental em pacientes com HIV. Revista Psicologia: Teoria e Prática. 17(2) 36-51. http://pepsic.bvsalud.org/scielo.php?script=sci_arttext\&pid=S151636872015000200003

Smith, C. (2003). Theorizing Religious Effects Among American Adolescents. Journal for the Scientiflc Study of Religion. 42(1), 17-30. https://csrs.nd.edu/assets/50016/theorizing_religious_effects_among_american_adolescents.pdf

Somefun, O. D. (2019). Religiosity and sexual abstinence among Nigerian youths: does parent religion matter? BMC Public Health. 19(416), 01-11. https://bmcpublichealth.biomedcentral.com/articles/10.1186/s12889-019-6732-2

Spindola, T., Teixeira, R. S., Antunes, R. F., \& Machado, Y. Y. (2020). Iniciação sexual e diálogo sobre sexualidade: visão de jovens universitários. Revista Recien. 10(30),106-116. https://www.recien.com.br/index.php/Recien/article/view/367/pdf

Teixeira, M. A. A. (2015). Provocações para uma sociologia da sexualidade: Sistemas, linguagem, amor. Revista Plural. 22(2), 182-203. http://www.revistas.usp.br/plural/article/view/112456/110408

Teixeira, R. S. (2016). As condutas sexuais dos jovens e o contexto das Infecções Sexualmente Transmissíveis: uma perspectiva de gênero. Dissertação (Mestrado em Enfermagem) - Faculdade de Enfermagem, Universidade do Estado do Rio de Janeiro, RJ.

World Health Organization. (2017). Sexual health [Internet]. WHO. http://www.scielo.br/pdf/tes/v17n2/1678-1007-tes-17-02- e0020233.pdf

Zerbinati, J. P., \& Toledo, M. A. B. (2017). Sexualidade e educação: revisão sistemática da literatura científica nacional. Travessias. 11(1), 76-92. http://saber.unioeste.br/index.php/travessias/article/view/16602/11276 